\title{
In-silico drug repurposing for targeting SARS-CoV-2 $\mathrm{M}^{\text {pro }}$
}

\author{
Shilpa Sharma, Shashank Deep* \\ Department of Chemistry, Indian Institute of Technology Delhi, Hauzkhas, New Delhi, 110016
}

\begin{abstract}
COVID-19, caused by novel coronavirus or SARS-CoV-2, is a viral disease which has infected millions worldwide. Considering the urgent need of the drug for fighting against this infectious disease, we performed in-silico drug repurposing. The main protease $\left(\mathrm{M}^{\mathrm{pro}}\right)$ is one of the best characterized drug targets among coronaviruses, therefore, this was screened for already known drugs, including chemical constituents of Ayurvedic drugs, using docking and MD simulation. The results suggest EGCG, withaferin A and artesunate may act as potential inhibitors of the main protease $\left(\mathrm{M}^{\mathrm{pro}}\right)$.
\end{abstract}

\section{Introduction}

A new coronavirus, named SARS-CoV-2, has started from Wuhan, China and spread over the world $^{1-3}$. It is a severe acute respiratory syndrome related coronavirus ${ }^{4}$. The coronavirus COVID19 has affected 210 countries and territories around the world, among them USA, Spain, Italy, France, Germany, UK and Turkey are the worst hit countries having over one million infections. As on April 28, 2020, around 30 million people have been infected, and 2.1 million have died ${ }^{5}$. The number of infected person is increasing exponentially and not a single vaccine or drug is available for treatment although few of them have shown the potential. Currently, affected patients are receiving antimalarial drugs chloroquine and hydroxychloroquine ${ }^{6,7}$ as treatment but recent data suggest that this may not help and side effects involved with these medications can be life- 
threatening. Development of vaccine is still one year away and there is a strong need for repurposing an already known drug which can inhibit their replication.

Coronaviruses are large family of RNA viruses which are encapsulated inside a membrane envelope. Envelop has proteins appearing like spikes sticking out from their surface ${ }^{8}$. Main protease (Mpro) is an enzyme involved in processing of polyprotein which is translated from viral RNA. Inhibition of this protease will block the replication of the virus and thus is an important drug target. The crystal structure of the Mpro of SARS-CoV-2 has been recently reported and alpha-ketomide based inhibitors have also been reported. One of the inhibitor has shown low micromolar EC-50 against SARS-CoV-2 ${ }^{9}$.

MPro is a homodimer, each monomer consisting of three domains. Dimerization of enzyme is necessary for catalytic activity since it helps in making of S1 pocket of substrate binding site ${ }^{10,11}$. Thus, any drug like candidate which strongly binds to S1 site or inhibit the dimerization process can potentially inhibit the replication of virus. Docking and MD simulation has emerged as a tool to predict the putative drugs if the target protein structure is available.

Recently several research groups have reported some inhibitors of SARS-CoV-2 main protease ${ }^{9,12-}$ 15. In our study, we have carried out in-silico screening of a range of FDA-approved antiviral, antimalarial drugs, some Ayurveda medicines and some natural polyphenols targeting Mpro. We have arranged obtained hits according to their interaction energies and top compounds have been subjected to molecular dynamics simulations in much more realistic environment.

\section{Materials and Methods:}

\section{Preparation of protein structure and ligand database for docking}

The crystal structure of the free enzyme of the SARS-CoV-2 main protease (PDB: 6Y2E) was recently published by Zhang and coworkers ${ }^{9}$. Its substrate binding pocket was chosen 
for the screening of compounds. The ligand database was prepared by retrieving the structures of FDA approved antiviral and antimalarial drugs from Drugbank ${ }^{16}$, structures $^{2}$ of Ayurveda compounds from Pubchem ${ }^{17}$ and structures of polyphenols were drawn using Marvin Sketch and minimized 3D structures were obtained ${ }^{18}$.

\section{Docking analysis}

AutoDock Vina ${ }^{19}$ was used for the screening of ligand database. AUTODOCK $4^{20}$ was used to prepare the ligand structure for docking. Docking generates several poses of ligand inside the binding pocket, the poses showing maximum interaction i.e. minimum binding energy were chosen for carrying out further studies. Various interactions like hydrophobic and hydrogen bonding interactions between ligand and protein were visualized using $\operatorname{LigPlot}^{21}$.

\section{Molecular dynamics simulations}

Molecular dynamics simulations were carried out on Amber18 package ${ }^{22}$. Ligand parameters were obtained from general Amber force field (GAFF2) ${ }^{23}$ and AM1 BCC method was used to derive the charges. Bonded and non-bonded parameters for protein were obtained from Amber 14SB force field. Docked protein-ligand complexes were solvated in a $10 \mathrm{~nm}$ truncated octahedron with TIP3P water molecules ${ }^{24}$ and counter ions were added to neutralize the system. The study utilized periodic boundary conditions and PME summation $^{25}$ for electrostatic calculations.

The shake methodology was applied to restrict covalently bonded hydrogen atoms. Constant pressure condition was maintained using Berendsen thermostat ${ }^{26}$. Time step of 2 femtosecond with $9 \mathrm{~A}^{0}$ cut off for non-bonded interactions were applied. Protein-ligand complexes were energy minimized in two steps, first using 250 steps of steepest descent 
followed by 750 steps of conjugate gradient method. Equilibration was also carried out in two steps, heating the systems at $300 \mathrm{~K}$ followed by simulation of complexes by decreasing the force slowly up to $0.1 \mathrm{~N}$. Next, fully unrestricted equilibration was performed for $5 \mathrm{~ns}$. Convergence of various system properties was monitored. Production run was performed for 500 ns under NPT ensemble. Simulation of protein without any ligand was also carried out.

\section{Analysis of MD simulation trajectory}

For analysis Amber trajectory was converted to Gromacs ${ }^{27}$ trajectory using python script. For checking the stability of protein due to ligand binding, root mean square deviation (RMSD) was monitored with respect to the reference structure (first frame) for $\mathrm{C}_{\alpha}$ atoms of protein. Clustering analysis was done was done by taking the last 100 ns trajectory, clustering was done using single linkage method. In this, a cluster was formed by subsequent addition of structures whose distance is less than $0.1 \mathrm{~nm}$ to any element of the cluster, if distance is more than $0.1 \mathrm{~nm}$ a new cluster was formed.

\section{Results}

\section{In-silico screening of Antiviral, Antimalarial drugs, Polyphenols and Ayurvedic} compounds through molecular docking with Mpro

In order to find the potential binders to the substrate site of Mpro (PDB: $6 \mathrm{Y}^{2} \mathrm{E}^{9}$ ), a total of 125 FDA approved drugs and compounds were screened utilizing molecular docking studies. Out of total, 31 compounds have binding energy below $-6 \mathrm{kcal} / \mathrm{mol}, 52$ compounds have binding energy between -6 to $-7 \mathrm{kcal} / \mathrm{mol}, 35$ compounds have binding energy between -7 to $-8 \mathrm{kcal} / \mathrm{mol}$ and only 7 compounds have binding energy above $-8 \mathrm{kcal} / \mathrm{mol}$ (Table S1). Compounds having binding energies above or equal to $-8 \mathrm{kcal} / \mathrm{mol}$ were 
selected for carrying out molecular dynamics simulation to understand their molecular level interactions with the receptor, their binding energy and interacting residues are listed in Table 1. The selected seven compounds were epigallocatechin gallate (EGCG), delavirdine, dolutegravir, indinavir, artesunate, tinosporin B and withaferin A .

Epigallocatechin gallate (EGCG) is a polyphenolic compound found in green tea and have been shown to have antiviral activity. The calculated binding energy between EGCG and Mpro from docking is $-8.3 \mathrm{kcal} / \mathrm{mol}$ and EGCG mainly interacts with residues Phe 140, Leu 141, Ser 144 and Glu 166 via hydrogen bonding interaction and with residues Thr 25,His 41,Ser 46,Met 49,Cys 145,Met 165 and Gln 189 via hydrophobic interactions (figure $3(\mathrm{c})$ ).

Delavirdine, dolutegravir and indinavir are FDA approved antiviral drugs used for the treatment of Human Immunodeficiency virus (HIV). Binding energies obtained for delavirdine, dolutegravir and indinavir are $-8.1 \mathrm{kcal} / \mathrm{mol},-8.6 \mathrm{kcal} / \mathrm{mol}$ and $-8.0 \mathrm{kcal} / \mathrm{mol}$, respectively. Delavirdine interacts with Phe 140 and Asn 142 through hydrogen bonding and residues Thr 25,Thr 26,Leu 27,His 41, Ser 46,Met 49, Leu 141, Gly 143, Cys 145,His 163 and Glu 166 are involved in hydrophobic interactions (figure $3(\mathrm{~g})$ ). Dolutegravir mainly interacts with Gly 143, Thr 24, Thr 25,His 41,Thr 45,Ser 46,Asn 142, Gly 143, Cys 145 and Glu 166 (figure 3 (i)). Similarly, residues Asn 142,Thr 25, Thr 26, His 41,Met 49,Phe 140,Leu 141,Asn 142,Cys 145,His 163,Glu 166 and Gln 189 interact with indinavir (figure $3(\mathrm{k})$ ). 
Artesunate is one of the most rapid acting antimalarial drugs and it is a semi-synthetic derivative of artemisinin. Calculated binding energy for protein-artesunate complex is -8 $\mathrm{kcal} / \mathrm{mol}$ and it shows interactions with residues Thr 24-26, Leu 27, His 41,Ser 46, Met 49,Phe 140,Leu 141,Asn 142,Gly 143,Ser 144,Cys 145,His 163,Met 165 and Gln 166 (figure $3(\mathrm{~m})$ ).

Next, two Ayurveda compounds also showed significant binding to the target, one is tinosporin $\mathrm{B}$ which is a diterpenoid furanolactones extracted from plant tinospora cardyfolia. Tinosporin has anti-inflammatory, anti-microbial, antihypertensive and antiviral properties. Tinosporin B exhibits interactions with residues Thr 25,His 41,Cys 44, Thr 45,Ser 46,Met 49,Asn 142,Cys 145,His 164,Met 165, Glu 166 and Gln 189 with $8.0 \mathrm{kcal} / \mathrm{mol}$ binding energy (figure 3 (a)). Another Ayurveda compound which qualified out binding energy criterion is withaferin A, it is extracted from the leaves of the Indian plant Withania somnifera and exhibits chemo preventive, anti-cancer and immunomodulatory actions. Withaferin A shows interactions with residues Thr 24,Thr 25,His 41,Cys 44,Ser 46,Met 49,Phe 140,Leu 141,Asn 142,His 164 and Glu 166 with binding energy equals to $-8.9 \mathrm{kcal} / \mathrm{mol}$ (figure 3 (e)). 
Table 1 Binding parameters and interacting residues for Mpro-ligand complexes obtained from docking simulations.

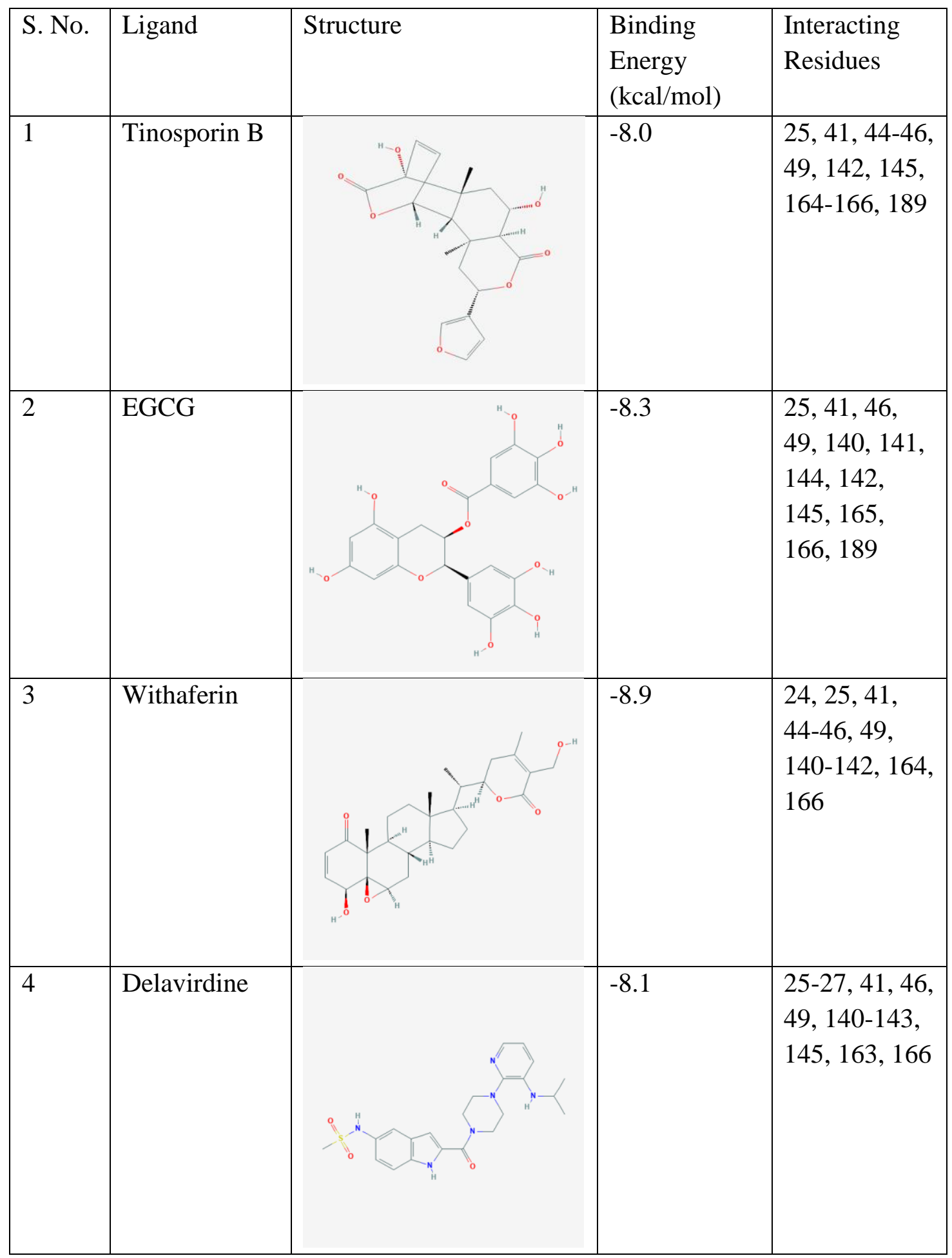




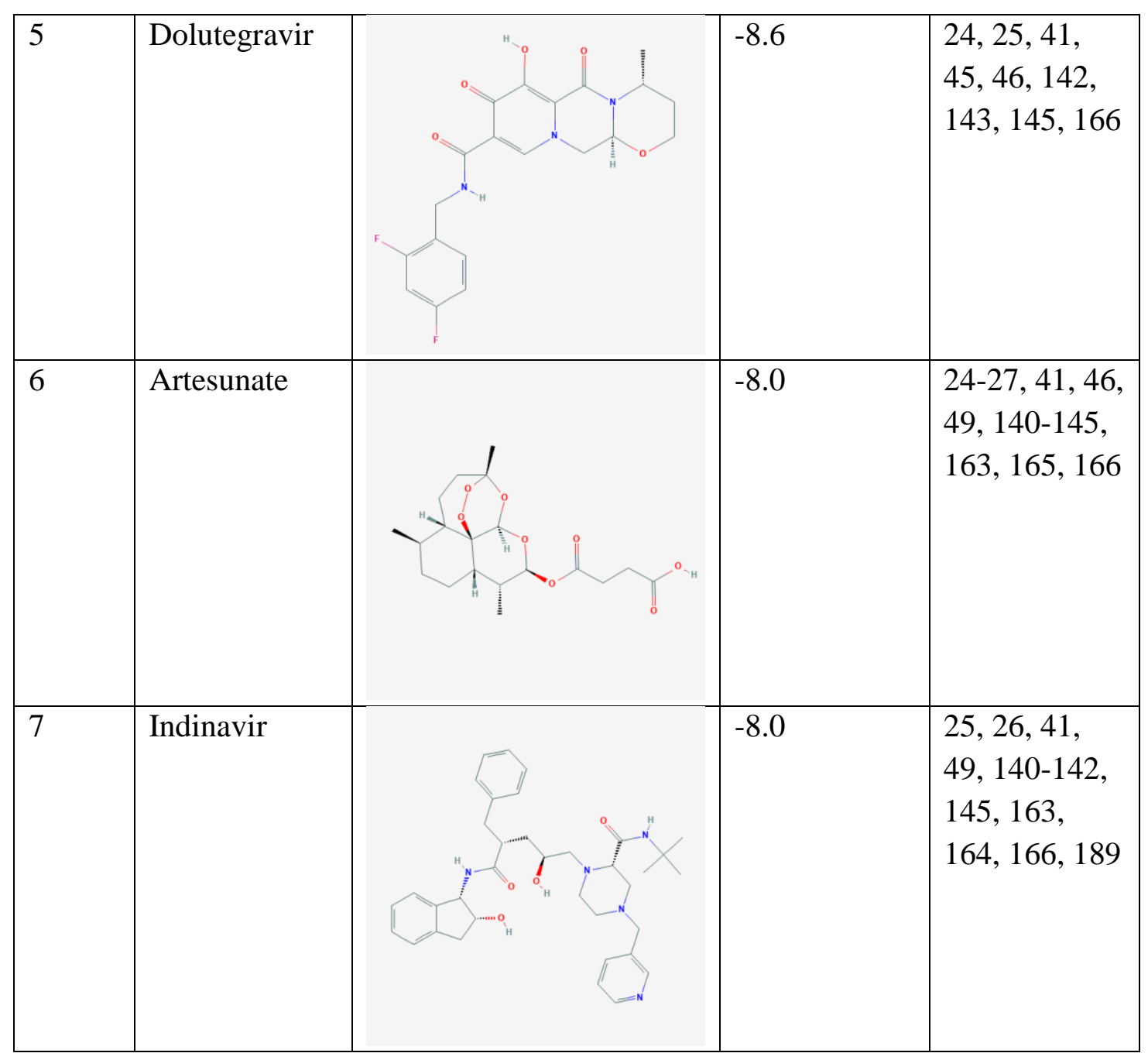

\section{Molecular Dynamics (MD) Simulations}

Molecular dynamics simulations were performed to ascertain the stability of docked complexes. Root mean square deviation (RMSD) values of $\mathrm{C}_{\alpha}$ were plotted with respect to the first frame for all the complexes (figure 1 (a)). For a better comparison average values of RMSD for last $100 \mathrm{~ns}$ were plotted along with standard deviation (figure 1 (b)). RMSD value for artesunate complex increased from $0.2589 \mathrm{~nm}( \pm 0.0165)$ in free protein to 0.6845 $\mathrm{nm}( \pm 0.1461)$, which indicated towards the structure fluctuation due to ligand binding. Least RMSD changes were observed for EGCG complex with a value of $0.1854 \mathrm{~nm}( \pm$ 
0.01641) showing the stable docked complex formation. For others RMSD values remain in the range of $0.2367-0.3702 \mathrm{~nm}$.
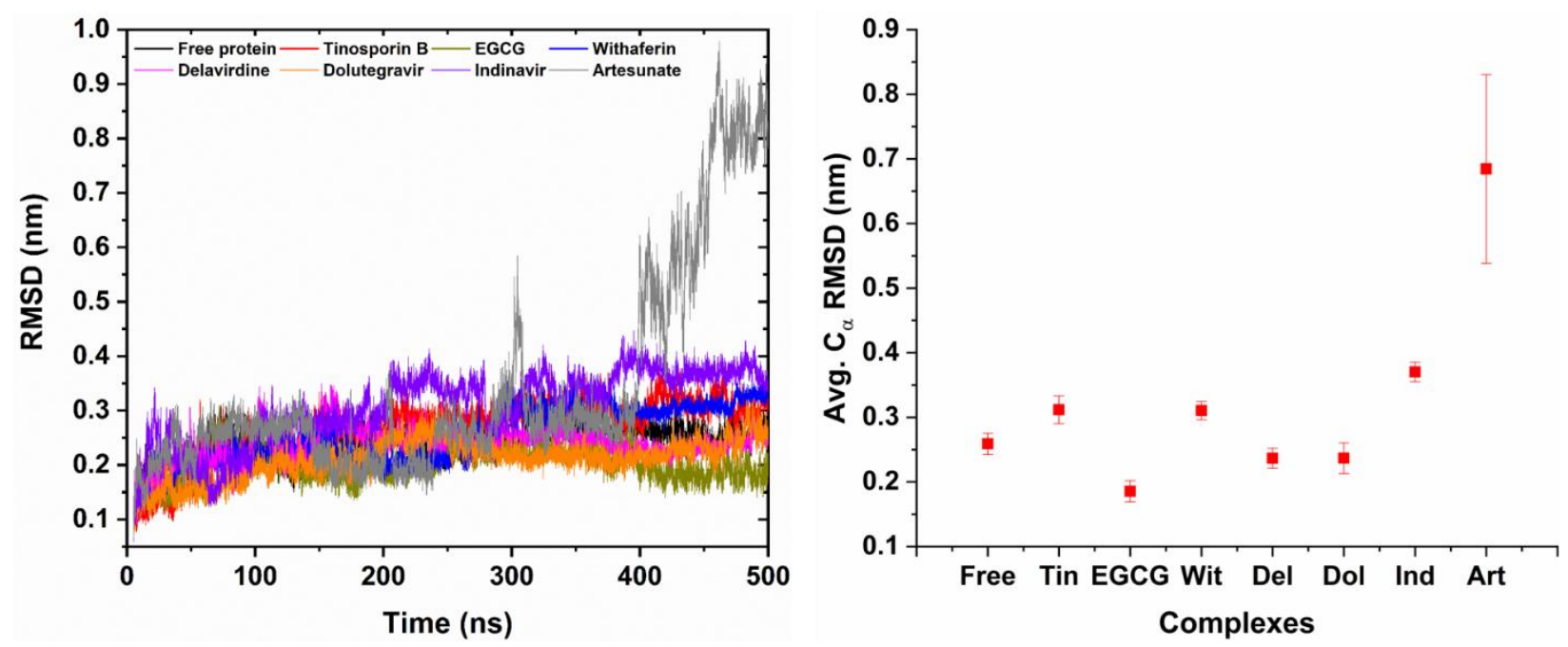

Figure 1 (a) $\mathrm{C}_{\alpha}$ root mean square deviation plot, (b) Average RMSD values for last $100 \mathrm{~ns}$. Next, clustering analysis was done on last 100 ns trajectory (figure 2) and a representative structure was obtained which then visualized through LigPlot to see the change in molecular level interaction during simulations (figure 3).

Maximum number of clusters were obtained for artesunate i.e. 110 with an average RMSD $0.3478 \mathrm{~nm}$. Delavirdine and withaferin showed minimum number of clusters, one in each case with an average RMSD $0.1353 \mathrm{~nm}$ and $0.1218 \mathrm{~nm}$, respectively. Tinosporin, dolutegravir and EGCG showed 2, 8 and 6 clusters with an average RMSD $0.1567 \mathrm{~nm}$, $0.1480 \mathrm{~nm}$ and $0.1338 \mathrm{~nm}$, respectively. A representative structure of the most populated 
cluster was obtained for all the complexes. When there are large number of clusters it is very difficult to obtain a very accurate depiction as in the case of artesunate.

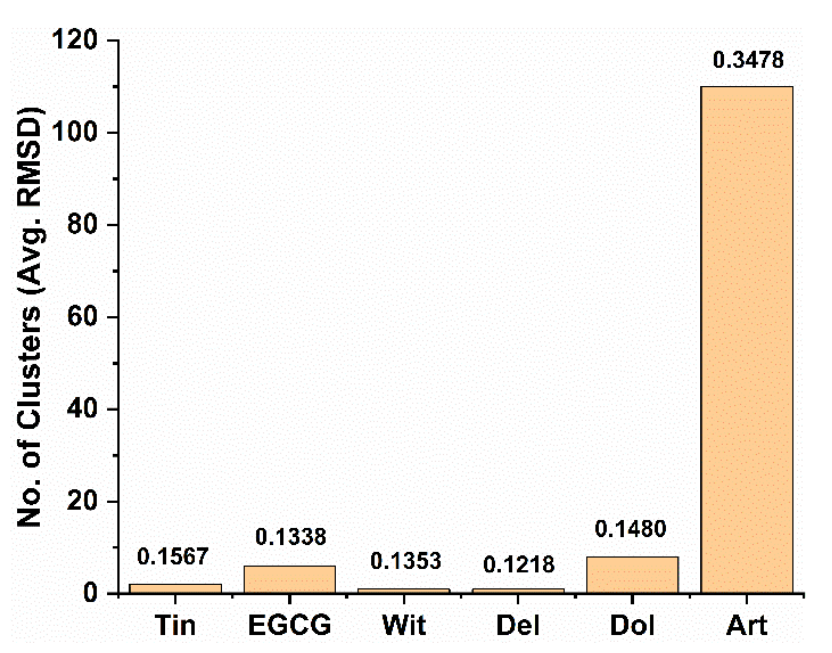

Figure 2 Clustering analysis on the basis of RMSD for last 100 ns trajectory.

Next, the obtained average structure from clustering analysis was compared with the docked structure before the start of simulation, for the conserved interactions (figure 3 (a)(n)). For tinosporin, dolutegravir and indinavir, no conserved interactions were seen, binding site for these ligands were changed completely. Delavirdine with one intact contact also showed weak binding interaction. For protein-EGCG complex, number of interactions were increased with five conserved interactions, indicating towards a good binding of ligand to its receptor. Withaferin showed two conserved interactions and also seen to be well within the desired binding pocket. Although, for artesunate larger number of clusters 
were obtained but the one conformation that occurs maximum number of time, exhibited eight number of conserved interactions. Therefore, on the basis of conserved interactions tinosporin. Dolutegravir, indinavir and delavirdine have ruled out to be tested as a drug candidate and EGCG, withaferin, artesunate and their analogs can be considered as good antidotes for COVID-19 and further studies can be carried out on them.

(a)

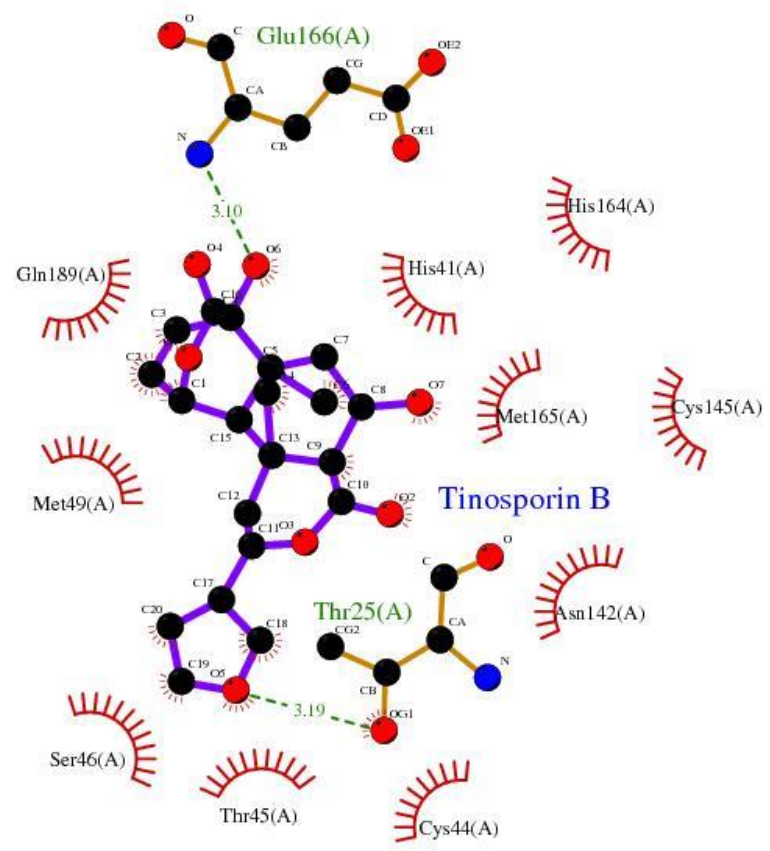

(b)

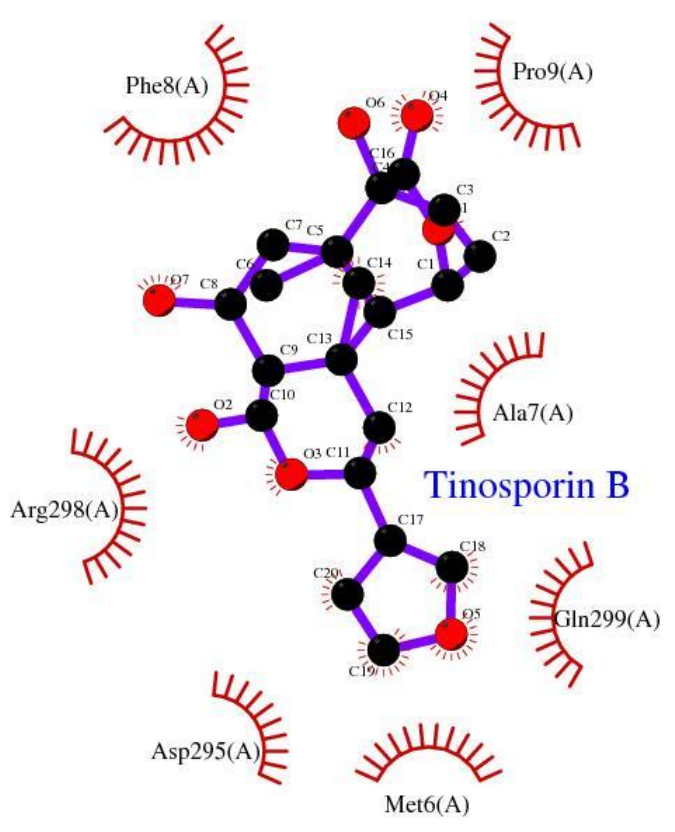


(c)

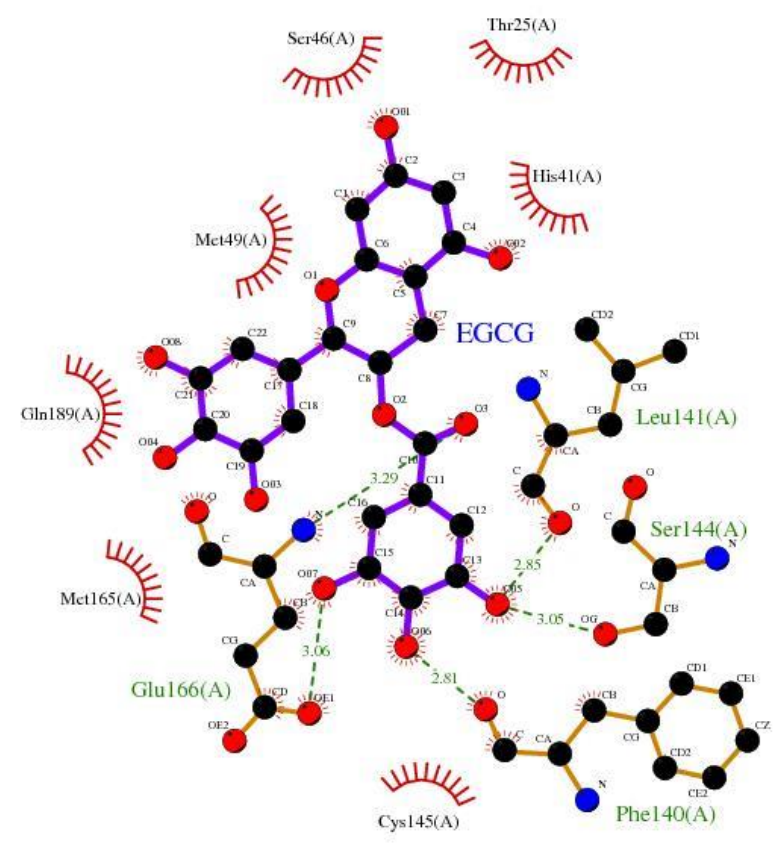

(e)

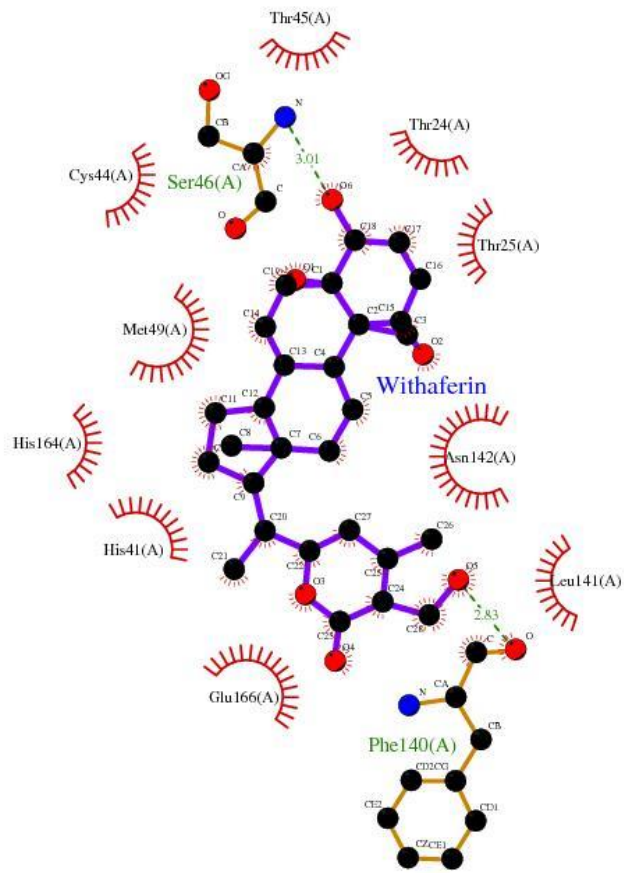

(d)

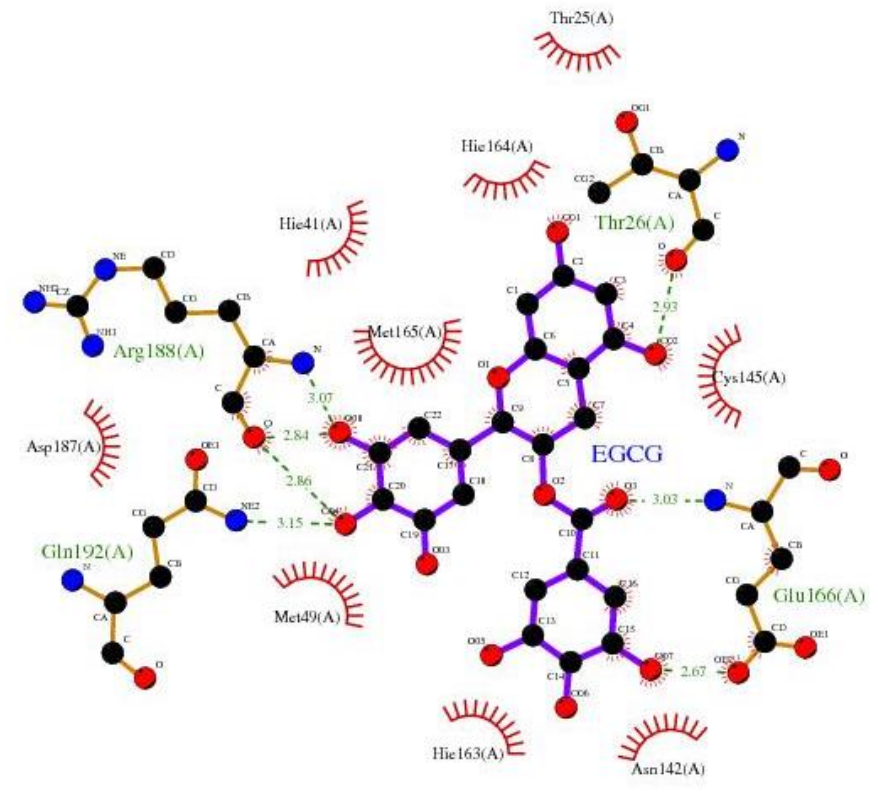

(f)

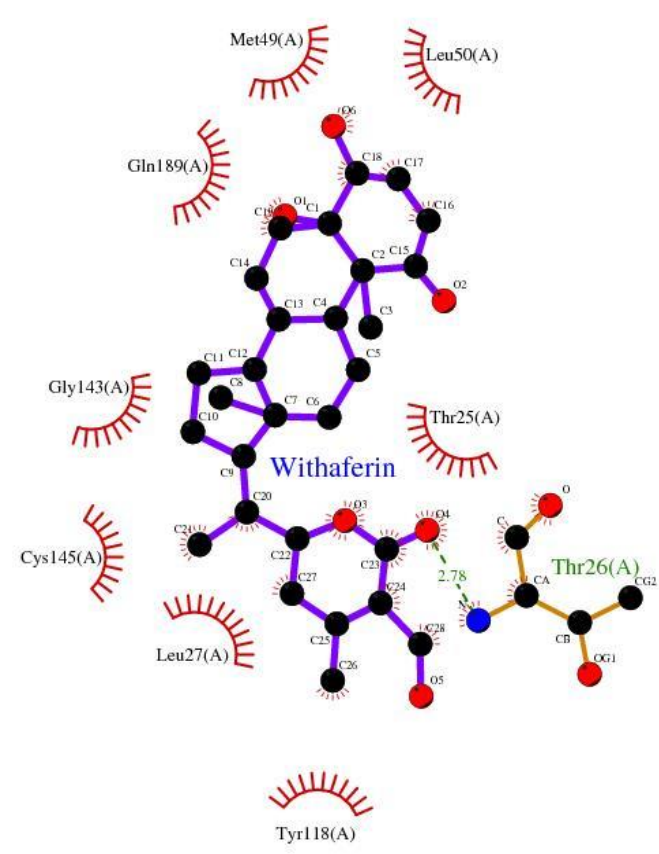


(g)

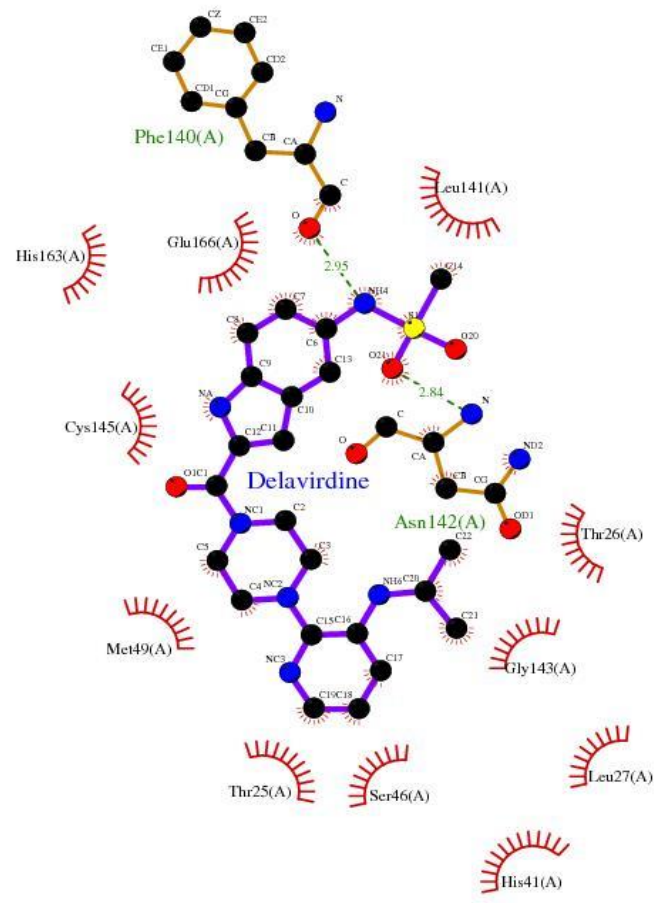

(i)

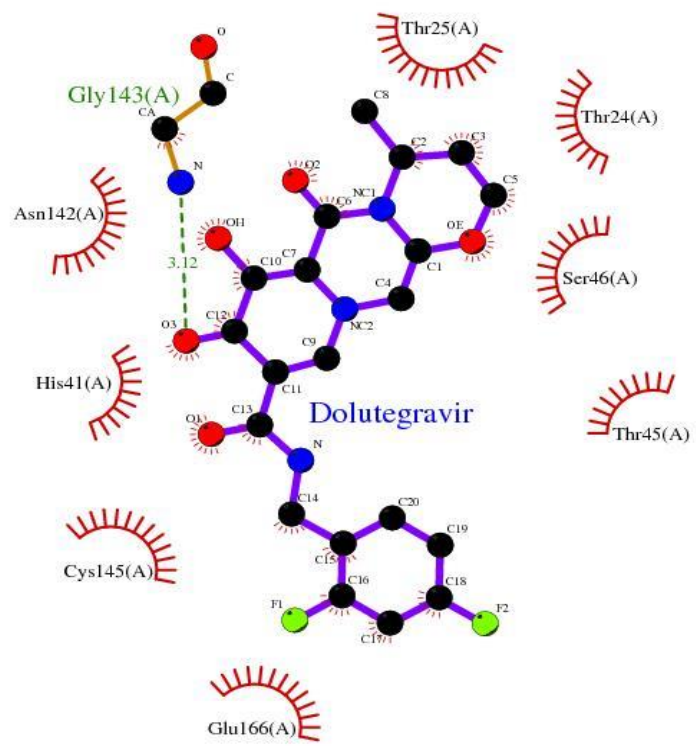

(h)

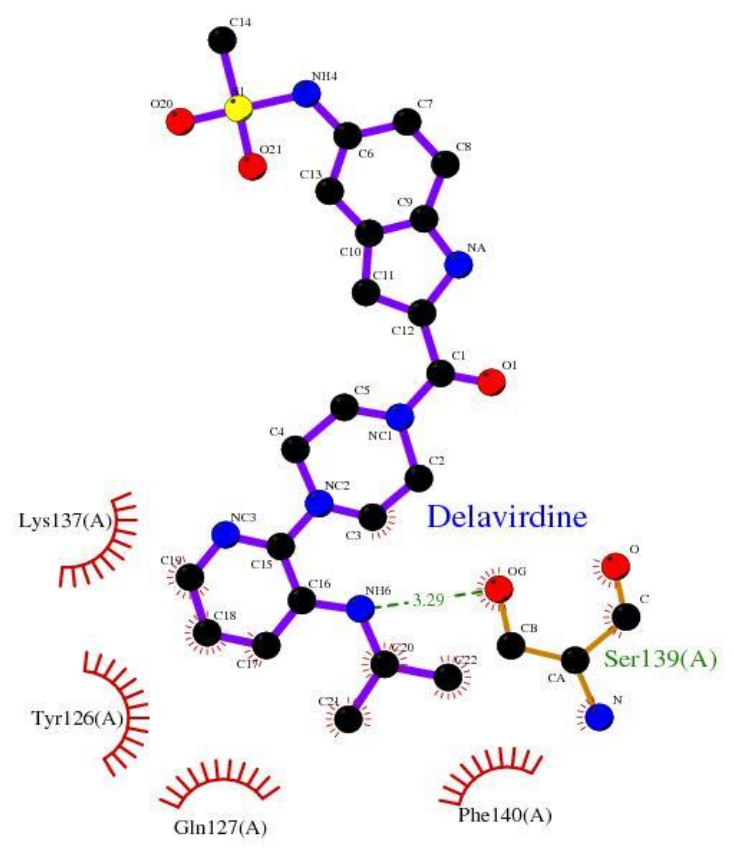

(j)

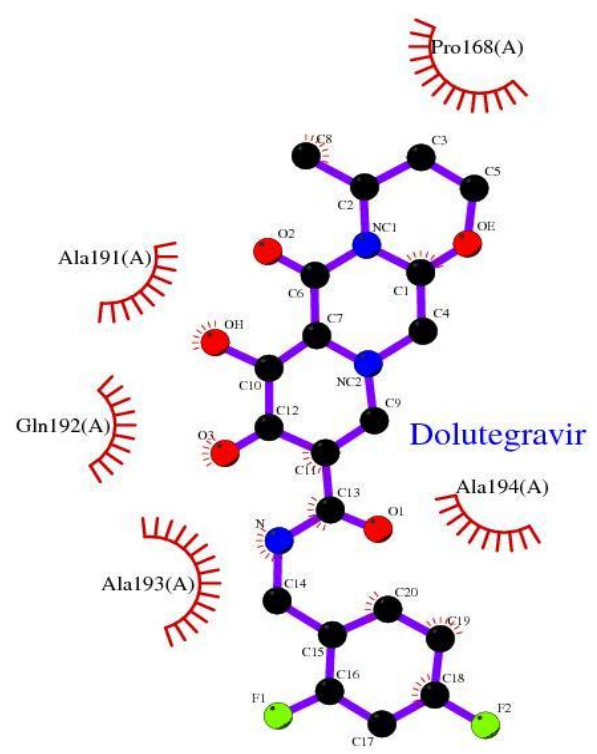


(k)

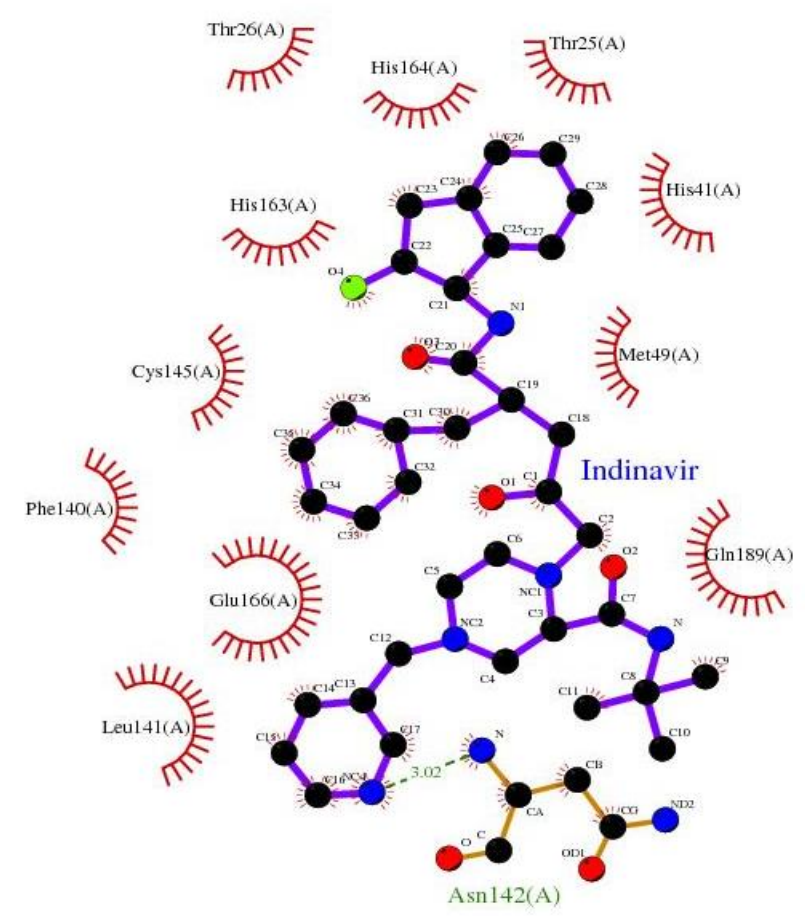

(m)

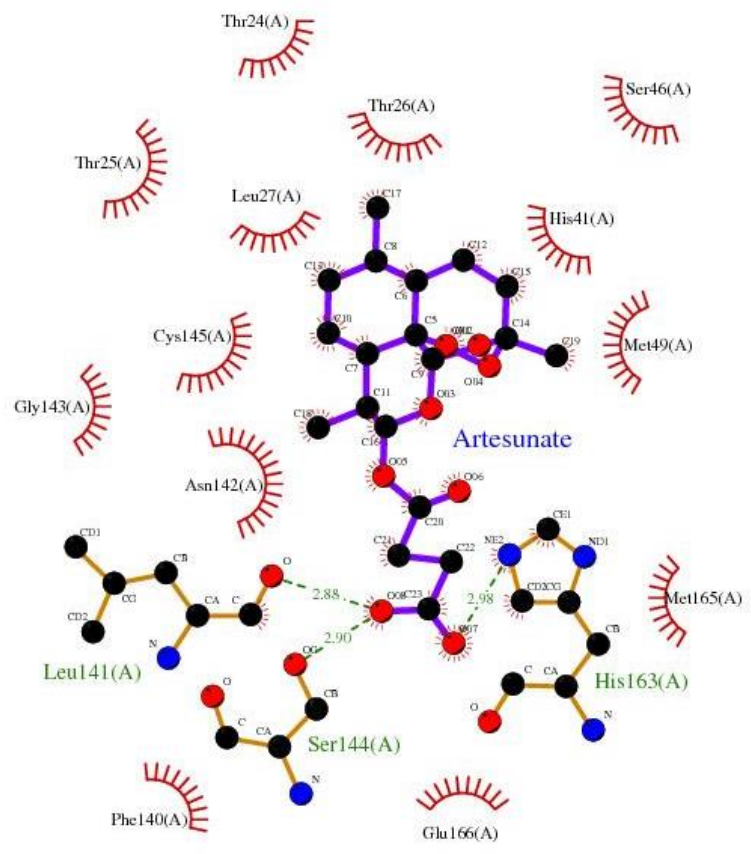

(I)

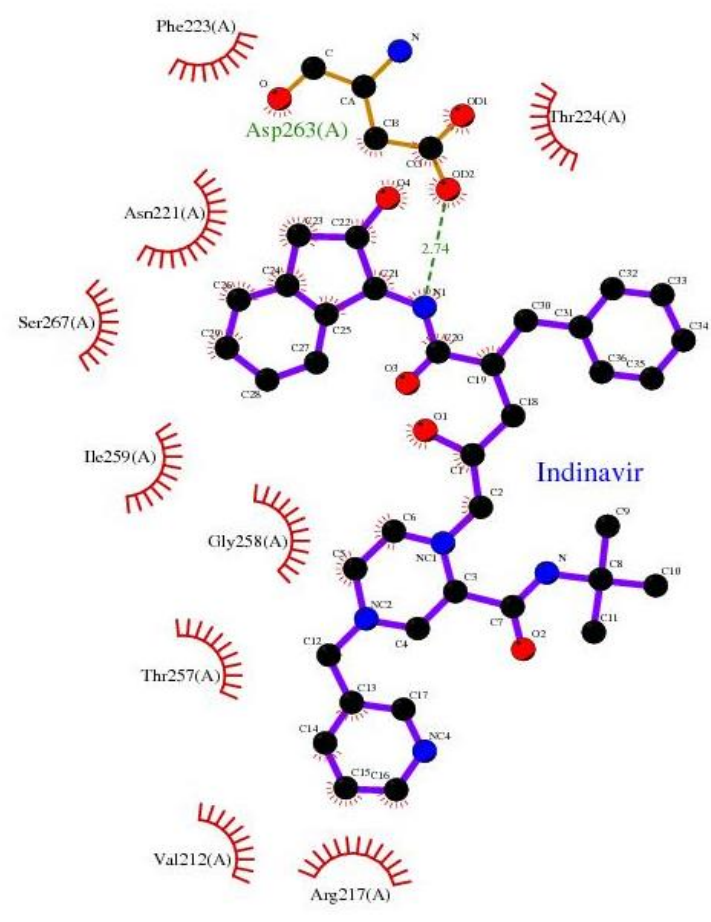

(n)

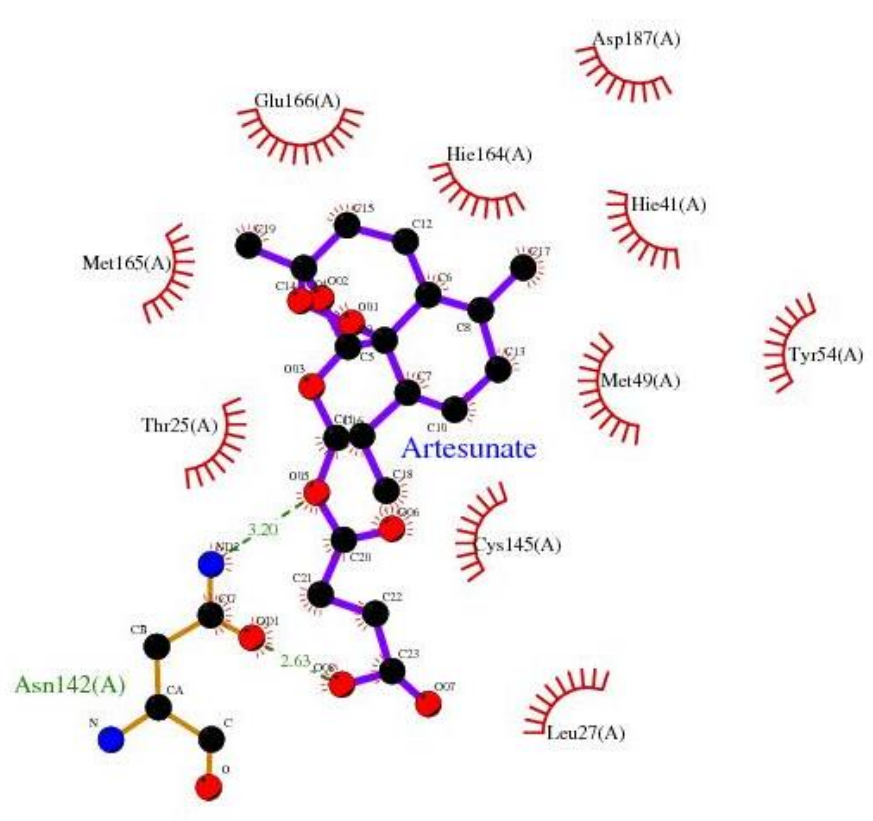


Figure 3 Interaction between SARS-CoV-2 Mpro and Tinosporin B; (a) before MD and (b) after MD or EGCG; (c) before MD and (d) after MD or Withaferin A; (e) before MD and (f) after MD or Delavirdine; (g) before MD and (h) after MD or Dolutegravir; (i) before MD and (j) after MD or Indinavir; (k) before MD and (l) after MD or Artesunate; (m) before MD and (n) after MD as observed from Ligplot. Green dashed lines represent hydrogen bonds and half-moons represents hydrophobic interactions. Residues in black font are involved in hydrophobic interactions and residues in green font are involved in hydrogen bonding.

\section{Conclusion}

Our docking and molecular dynamics suggests EGCG, withaferin A and artesunate and their analogs may act as inhibitor for MPro protease of SARS-CoV-2. EGCG which is a major component of green tea is capable of showing antiviral activity against many DNA and RNA viruses, like adenovirus, influenza virus, human immunodeficiency virus (HIV) and coronavirus, by inhibiting various stages of viral infection ${ }^{28-32}$. Similarly, withaferin which is a component of ayurvedic plant Withania somnifera also exhibits antiviral properties again influenza virus ${ }^{33}$. Artesunate, a semisynthetic derivative of artemisinin, is a well-known malaria drug and has potential to fight against many viruses ${ }^{34,35}$. Supported by previous literature and our results we propose EGCG, withaferin A and artesunate as potential drugs for COVID-19. Further, experimental work needs to be done to verify their use as a drug. 


\section{Associated Content}

\section{Supporting Information}

Supplementary Table S1.

Author Information

Corresponding Author

*Shashank Deep, Professor

Department of Chemistry, Indian Institute of Technology Delhi, New Delhi, 110016, India.

E-mail: sdeep@chemistry.iitd.ac.in

\section{Acknowledgements}

SS thank University Grants Commission (UGC) for financial support. We thank IIT Delhi HPC facility for computational resources. We acknowledge Supercomputing Facility for Bioinformatics and Computational Biology (SCFBio) IIT Delhi for granting the access of AMBER.

\section{Conflict of interest}

There is no conflict of interest. 


\section{References:}

(1) Wu, F.; Zhao, S.; Yu, B.; Chen, Y.-M.; Wang, W.; Song, Z.-G.; Hu, Y.; Tao, Z.-W.; Tian, J.-H.; Pei, Y.-Y.; et al. A New Coronavirus Associated with Human Respiratory Disease in China. Nature 2020, 579 (7798), 265-269. https://doi.org/10.1038/s41586-020-2008-3.

(2) Shereen, M. A.; Khan, S.; Kazmi, A.; Bashir, N.; Siddique, R. COVID-19 Infection: Origin, Transmission, and Characteristics of Human Coronaviruses. J. Adv. Res. 2020, 24, 91-98. https://doi.org/10.1016/j.jare.2020.03.005.

(3) Zhou, P.; Yang, X.-L.; Wang, X.-G.; Hu, B.; Zhang, L.; Zhang, W.; Si, H.-R.; Zhu, Y.; Li, B.; Huang, C.-L.; et al. A Pneumonia Outbreak Associated with a New Coronavirus of Probable Bat Origin. Nature 2020, 579 (7798), 270-273. https://doi.org/10.1038/s41586020-2012-7.

(4) The Species Severe Acute Respiratory Syndrome-Related Coronavirus: Classifying 2019NCoV and Naming It SARS-CoV-2. Nat. Microbiol. 2020, 5 (4), 536-544. https://doi.org/10.1038/s41564-020-0695-z.

(5) Worldometers.info. 28 April, 2020, Dover, Delaware, U.S.A.

(6) Principi, N.; Esposito, S. Chloroquine or Hydroxychloroquine for Prophylaxis of COVID19. Lancet Infect. Dis. 2020. https://doi.org/10.1016/S1473-3099(20)30296-6.

(7) Singh, A. K.; Singh, A.; Shaikh, A.; Singh, R.; Misra, A. Chloroquine and Hydroxychloroquine in the Treatment of COVID-19 with or without Diabetes: A Systematic Search and a Narrative Review with a Special Reference to India and Other Developing Countries. Diabetes Metab. Syndr. 2020, 14 (3), 241-246. https://doi.org/10.1016/j.dsx.2020.03.011.

(8) Walls, A. C.; Park, Y.-J.; Tortorici, M. A.; Wall, A.; McGuire, A. T.; Veesler, D. Structure, Function, and Antigenicity of the SARS-CoV-2 Spike Glycoprotein. Cell 2020, 181 (2), 281-292.e6. https://doi.org/https://doi.org/10.1016/j.cell.2020.02.058.

(9) Zhang, L.; Lin, D.; Sun, X.; Curth, U.; Drosten, C.; Sauerhering, L.; Becker, S.; Rox, K.; Hilgenfeld, R. Crystal Structure of SARS-CoV-2 Main Protease Provides a Basis for Design of Improved $\alpha$-Ketoamide Inhibitors. Science. 2020, 368 (6489), 409 LP - 412. https://doi.org/10.1126/science.abb3405.

(10) Jin, Z.; Du, X.; Xu, Y.; Deng, Y.; Liu, M.; Zhao, Y.; Zhang, B.; Li, X.; Zhang, L.; Peng, C.; et al. Structure of M(pro) from COVID-19 Virus and Discovery of Its Inhibitors. Nature 2020. https://doi.org/10.1038/s41586-020-2223-y.

(11) Anand, K.; Ziebuhr, J.; Wadhwani, P.; Mesters, J. R.; Hilgenfeld, R. Coronavirus Main Proteinase (3CLpro) Structure: Basis for Design of Anti-SARS Drugs. Science 2003, 300 (5626), 1763-1767. https://doi.org/10.1126/science.1085658.

(12) Verma, D.; Kapoor, S.; Das, S.; Thakur, K. Potential Inhibitors of SARS-CoV-2 Main 
Protease (Mpro) Identified from the Library of FDA Approved Drugs Using Molecular Docking Studies. Preprints 2020, 202004.

https://doi.org/10.20944/preprints202004.0149.v1).

(13) Adem, S.; Eyupoglu, V.; Sarfraz, I.; Rasul, A.; Ali, M. Identification of Potent COVID-19 Main Protease (Mpro) Inhibitors from Natural Polyphenols: An in Silico Strategy Unveils a Hope against CORONA. Preprints 2020, 202003. https://doi.org/10.20944/preprints202003.0333.v1.

(14) Khan, S. A.; Zia, K.; Ashraf, S.; Uddin, R.; Ul-Haq, Z. Identification of Chymotrypsinlike Protease Inhibitors of SARS-CoV-2 via Integrated Computational Approach. $J$. Biomol. Struct. Dyn. 2020, 1-10. https://doi.org/10.1080/07391102.2020.1751298.

(15) Ton, A.-T.; Gentile, F.; Hsing, M.; Ban, F.; Cherkasov, A. Rapid Identification of Potential Inhibitors of SARS-CoV-2 Main Protease by Deep Docking of 1.3 Billion Compounds. Mol. Inform. 2020.

(16) Wishart DS, Knox C, Guo AC, Shrivastava S, Hassanali M, Stothard P, Chang Z, W. J. Drugbank: a comprehensive resource for in silico drug discovery and exploration. Nucleic Acids Res. 2006 Jan 1;34 (Database issue):D668-72. 16381955.

(17) Kim S, Chen J, C. T. PubChem 2019 Update: Improved Access to Chemical Data. Nucleic Acids Res. 2019;47(D1)D1102-D1109. https://doi.org/10.1093/nar/gky1033.

(18) Marvin Was Used for Drawing the Chemical Structures, MarvinSketch 17.23.0, ChemAxon (https://www.chemaxon.com).

(19) Trott, O.; Olson, A. J. AutoDock Vina: Improving the Speed and Accuracy of Docking with a New Scoring Function, Efficient Optimization, and Multithreading. J. Comput. Chem. 2010, 31 (2), 455-461. https://doi.org/10.1002/jcc.21334.

(20) Morris, G. M.; Huey, R.; Lindstrom, W.; Sanner, M. F.; Belew, R. K.; Goodsell, D. S.; Olson, A. J. AutoDock4 and AutoDockTools4: Automated Docking with Selective Receptor Flexibility. J. Comput. Chem. 2009, 30 (16), 2785-2791. https://doi.org/10.1002/jcc.21256.

(21) Laskowski, R. A.; Swindells, M. B. LigPlot+: Multiple Ligand-Protein Interaction Diagrams for Drug Discovery. J. Chem. Inf. Model. 2011, 51 (10), 2778-2786. https://doi.org/10.1021/ci200227u.

(22) D.A. Case, I.Y. Ben-Shalom, S.R. Brozell, D.S. Cerutti, T.E. Cheatham, III, V.W.D. Cruzeiro, T.A. Darden, R.E. Duke, D. Ghoreishi, M.K. Gilson, H. Gohlke, A.W. Goetz, D. Greene, R Harris, N. Homeyer, S. Izadi, A. Kovalenko, T. Kurtzman, T.S. Lee, S. LeGra, D. M. Y. and P. A. K. (2018) AMBER 2018. University of California: San Francisco .

(23) Träg, J.; Zahn, D. Improved GAFF2 Parameters for Fluorinated Alkanes and Mixed Hydro- and Fluorocarbons. J. Mol. Model. 2019, 25 (2), 39.

https://doi.org/10.1007/s00894-018-3911-5.

(24) Jorgensen, W. L.; Chandrasekhar, J.; Madura, J. D.; Impey, R. W.; Klein, M. L. 
Comparison of Simple Potential Functions for Simulating Liquid Water. J. Chem. Phys. 1983, 79 (2), 926-935. https://doi.org/10.1063/1.445869.

(25) Petersen, H. G. Accuracy and Efficiency of the Particle Mesh Ewald Method. J. Chem. Phys. 1995, 103 (9), 3668-3679. https://doi.org/10.1063/1.470043.

(26) Berendsen, H. J. C.; Postma, J. P. M.; van Gunsteren, W. F.; DiNola, A.; Haak, J. R. Molecular Dynamics with Coupling to an External Bath. J. Chem. Phys. 1984, 81 (8), 3684-3690. https://doi.org/10.1063/1.448118.

(27) Abraham, M. J., Van der Spoel, D., Lindahl, E., and Hess, B. No Title. GROMACS User Man. version 5.1.1; www.gromacs.org.

(28) Song, J.-M.; Lee, K.-H.; Seong, B.-L. Antiviral Effect of Catechins in Green Tea on Influenza Virus. Antiviral Res. 2005, 68 (2), 66-74. https://doi.org/10.1016/j.antiviral.2005.06.010.

(29) Weber, J. M.; Ruzindana-Umunyana, A.; Imbeault, L.; Sircar, S. Inhibition of Adenovirus Infection and Adenain by Green Tea Catechins. Antiviral Res. 2003, 58 (2), 167-173. https://doi.org/10.1016/s0166-3542(02)00212-7.

(30) Nance, C. L.; Siwak, E. B.; Shearer, W. T. Preclinical Development of the Green Tea Catechin, Epigallocatechin Gallate, as an HIV-1 Therapy. J. Allergy Clin. Immunol. 2009, 123 (2), 459-465. https://doi.org/10.1016/j.jaci.2008.12.024.

(31) Ho, H.-Y.; Cheng, M.-L.; Weng, S.-F.; Leu, Y.-L.; Chiu, D. T.-Y. Antiviral Effect of Epigallocatechin Gallate on Enterovirus 71. J. Agric. Food Chem. 2009, 57 (14), 61406147. https://doi.org/10.1021/jf901128u.

(32) Benelli, R.; Venè, R.; Bisacchi, D.; Garbisa, S.; Albini, A. Anti-Invasive Effects of Green Tea Polyphenol EpiGalloCatechin-3-Gallate (EGCG), a Natural Inhibitor of Metallo and Serine Proteases. Biol. Chem. 2002, 383 (1), 101-105. https://doi.org/https://doi.org/10.1515/BC.2002.010.

(33) Cai, Z.; Zhang, G.; Tang, B.; Liu, Y.; Fu, X.; Zhang, X. Promising Anti-Influenza Properties of Active Constituent of Withania Somnifera Ayurvedic Herb in Targeting Neuraminidase of H1N1 Influenza: Computational Study. Cell Biochem. Biophys. 2015, 72 (3), 727-739. https://doi.org/10.1007/s12013-015-0524-9.

(34) Lisewski, A. M.; Quiros, J. P.; Ng, C. L.; Adikesavan, A. K.; Miura, K.; Putluri, N.; Eastman, R. T.; Scanfeld, D.; Regenbogen, S. J.; Altenhofen, L.; et al. Supergenomic Network Compression and the Discovery of EXP1 as a Glutathione Transferase Inhibited by Artesunate. Cell 2014, 158 (4), 916-928. https://doi.org/10.1016/j.cell.2014.07.011.

(35) Efferth, T.; Romero, M. R.; Wolf, D. G.; Stamminger, T.; Marin, J. J. G.; Marschall, M. The Antiviral Activities of Artemisinin and Artesunate. Clin. Infect. Dis. 2008, 47 (6), 804-811. https://doi.org/10.1086/591195. 